\title{
Antecedentes de un expolio: testimonios británicos sobre la primera retirada del rey José de Madrid (julio-diciembre 1808)
}

Rocío Coletes Laspra

Universidad de Oviedo ${ }^{1}$

CES.XVIII, núm. 21 (2011), págs. 35-58.

1 Desde diciembre de 2010 la autora es beneficiaria de una beca de formación de profesorado universitario (Departamento de Historia del Arte y Musicología, Universidad de Oviedo). Resolución publicada en el Bö A-2010-15573, 11 de octubre de 2010. 
Resumen: El presente artículo tiene por objeto aportar nuevos datos sobre el expolio sufrido en España durante la guerra de la independencia (1808-1814). A lo largo del trabajo se presenta y analiza una serie de documentos inéditos, conservados en archivos británicos, que dan constancia de un primer intento de saqueo protagonizado por el rey José Bonaparte, y que presenta la peculiaridad de haberse llevado a cabo al comienzo mismo de su reinado, en 1808.

Palabras clave: Guerra de la Independencia. José Bonaparte. Expolio artístico. Colección real española. Documentos de agentes británicos. 
La guerra de la independencia española y sus consecuencias, por lo que al patrimonio artístico se refiere, han sido objeto de estudio desde hace varias décadas. Los trabajos más destacados en relación con este episodio histórico son los de Ilse Hemel Lipschutz², Véronique Gérard-Powell ${ }^{3}$ y María Dolores Antigüedad del Castillo-Olivares ${ }^{4}$. En los tres casos se trata de estudios relacionados con el reinado de José Bonaparte en España y las implicaciones artísticas, nacionales e internacionales que supuso la presencia francesa en la Península. Durante los últimos años, el interés por este episodio histórico se ha visto incrementado gracias a la celebración del bicentenario de los acontecimientos y batallas ocurridas durante los años de ocupación napoleónica de España y Portugal. El expolio perpetrado durante esta época, precozmente estudiado por Juan Antonio Gaya Nuño ${ }^{5}$, se conoce más y mejor gracias a la contribución de personalidades como Susan Jenkins 6 , cuyo estudio se centra en la llegada al Reino Unido del botín interceptado en Vitoria por el duque de Wellington en 1813, botín también conocido, a partir de la obra homónima de Galdós ${ }^{7}$, como «el equipaje del rey José».

El presente artículo se inserta en este contexto historiográfico y de aportación documental sobre el reinado de José Bonaparte, sobre todo respecto al año 1808 y las consecuencias que tuvo la ocupación francesa para el patrimonio nacional. El conde de Toreno ya adelantaba en su voluminosa obra sobre la contienda española que el expolio imperial comenzó desde el inicio de la ocupación, afirmando que, entre otros, el comandante Joachim Murat, gobernador de Madrid durante algunos meses en 1808, fue uno de los iniciadores de la práctica del saqueo ${ }^{8}$. En este artículo se presenta una serie de documentos inéditos, y

2 Ilse Hemel Lipschutz, Spanish painting and the French Romantics, Cambridge (Ma.), Harvard University Press, 1972.

3 Véronique GÉrard-PowelL, «Joseph Bonaparte et la collection des Bourbons d'Espagne», en Études d'histoire de l'art en l'honneur d'Antoine Schnapper, París, Flammarion, 1998, págs. 408-414.

4 María Dolores Antigüedad del Castillo Olivares, El patrimonio artístico de Madrid durante el gobierno intruso, Madrid, Universidad de Educación a Distancia, 1999.

5 Juan Antonio GaYa Nuño, La pintura española fuera de España, Madrid, Espasa-Calpe, 1958.

6 Susan Jenkins, «Spanish "Gift” at Apsley House», English Heritage Historical Review (Cambridge), vol. 2 (2007), págs. 112-127.

7 Benito Pérez Galdós, El equipaje del rey José, 1875, Madrid, Hernando, 1969.

8 José María Queipo de Llano, conde de Toreno, Historia del levantamiento, guerra y revolución de España, 1848, Oviedo, Junta General del Principado de Asturias, 2009, vol. 2, págs. 499-500. 
hasta ahora desconocidos, que añaden más responsables a la lista de los beneficiarios de la rapiña francesa del primer año de ocupación, entre otros al propio José Bonaparte. Se analizará el contenido de un total de doce manuscritos en lengua inglesa, cuya trascripción ${ }^{9}$ constituye la base documental para el objetivo de este estudio: aportar datos que demuestran cómo, desde su llegada al trono de España, José Bonaparte tenía la clara intención de apropiarse de parte del patrimonio nacional.

\section{El primer año del reinado de José I Bonaparte}

El reinado de José Bonaparte en España comenzó con la abdicación de los Borbones a favor de Napoleón Bonaparte, «emperador de los franceses» desde 1804. Con el Tratado de Fontainebleau (17 de octubre de 1807) se facilitaba la entrada de las tropas napoleónicas en España so pretexto de invadir Portugal, fiel aliado del Reino Unido, cinco meses antes de la caída del favorito de Carlos IV, Manuel Godoy. La inestabilidad política existente en España culminaba con el Motín de Aranjuez (marzo de 1808) y las consecuentes destitución de Godoy y renuncia de Carlos IV, seguidas del primer reinado (19 de marzo-5 de mayo de 1808) de Fernando VII. Napoleón se las ingenió para hacer trasladar a Francia a la práctica totalidad de los miembros de la familia real española. El día 10 de mayo de 1808 se firmaba el Tratado de Bayona, en virtud del cual la dinastía borbónica española renunciaba al trono y otorgaba sus derechos a Napoleón, al tiempo que sus miembros aceptaban el exilio ${ }^{10}$.

Aplicando Napoleón su método recurrente de colocar al frente de un país conquistado — pacíficamente o no- a miembros de su propia familia, José Bonaparte, rey de Nápoles, pasó a ser José I, rey de España y de las Indias, el 4 de junio de 1808. El 28 de julio, solamente ocho días después de instalarse en el Palacio Real de Madrid ${ }^{11}$, el nuevo rey decidía evacuar esta ciudad tras enterarse de la derrota francesa en la batalla de Bailén, primer fracaso en la

9 La transcripción de los documentos y su correspondiente traducción por la autora de este artículo se encuentran al final del mismo.

10 Antonio Moliner, «La España a finales del siglo XVIII y la crisis de 1808», en La guerra de la independencia en España (1808-1814), Barcelona, Nabla, 2007, págs. 41-71 (pág. 53). El número 48 de la ya francesa Gazeta de Madrid publicaría el documento de abdicación el día 20 de mayo de 1808 en sus páginas 482-484.

11 Juan José LunA, «El Rey José», en La alianza de dos monarquías: Wellington en España (cat. expo. Museo Municipal de Madrid, oct.-dic. 1988), Madrid, Fundación Hispano-Británica, 1988, págs. 197-245 (pág. 210). 
Península del ejército imperial ${ }^{12}$. José había llegado a la capital el día 20 de julio, fecha en que la gran revuelta española contra las fuerzas extranjeras, cuyo detonante principal se sitúa en los alzamientos del 2 de mayo, había alcanzado dimensiones insospechadas. El rey intruso salió de Madrid el día 1 de agosto para concentrar sus fuerzas al norte del río Ebro, teniendo como centro de operaciones la ciudad de Vitoria ${ }^{13}$. El día 9 del mismo mes llegó a Burgos, y el 17 a Miranda de Ebro, ciudades sometidas al control del mariscal Moncey desde hacía varios meses. Más adelante el monarca y su séquito se trasladaron a Vitoria, convirtiéndose esta ciudad en la corte josefina hasta el mes de diciembre. José se mantuvo desde finales de agosto entre Miranda de Ebro, Bilbao y Vitoria, hasta que se produjo la llegada de Napoleón a esta última ciudad el día 5 de noviembre de $1808^{14}$.

Tras varias ofensivas del ejército imperial, que había sumado efectivos a lo largo de los meses precedentes, el día 4 de diciembre la capital española capitulaba ante Napoleón, quien entraría en Madrid junto a su hermano José el día 20 del mismo mes. José decidió entonces instalarse en el Palacio del Pardo, a las afueras de la urbe. Napoleón tuvo en ese momento la oportunidad de contemplar en los Reales Sitios los bienes acumulados por la monarquía española a lo largo de los siglos. La sorpresa que le produjo verse rodeado de tamañas obras maestras le hizo exclamar «en vérité, mon frère, vous êtes mieux logé que moi» ${ }^{15}$. José, por su parte, volvió a Madrid a finales de enero de 1809, y retomó su reinado a la vez que definía las características de su administración según el modelo planteado en la Constitución de Bayona de $1808^{16}$.

Es en este contexto de idas y venidas donde se insertan los documentos que aquí se dan a conocer. Se trata de un total de doce despachos que registran la correspondencia dirigida por varios agentes y oficiales del ejército británico a sus superiores del War Office, en los que se da constancia de las intenciones de José I de llevarse consigo una gran parte de los bienes de la corona española.

\footnotetext{
12 Manuel Moreno Alonso, José Bonaparte: un rey republicano en el trono de España, Madrid, La Esfera de los Libros, 2008, pág. 295.

13 Andrés Cassinello Pérez, «Evolución de las campañas militares», en Moliner, La guerra de la independencia, op. cit., págs. 73-122 (pág. 90).

14 Manuel Moreno Alonso, José Bonaparte, op. cit., págs. 299-300.

15 Jeannine Baticle, «La mission en Espagne», en Les vies de Dominique Vivant-Denon (actas de coloquio, Museo del Louvre, 8-11 dic. 1999), París, Museo del Louvre, 2001, vol. I, págs. 325-344 (pág. 328).

16 María Dolores Antigüedad del Castillo, El patrimonio artístico, op. cit., págs. 15-16.
} 
La intervención de Gran Bretaña en la que allí se conoce como The Peninsular War fue determinante para la victoria española. Las juntas provinciales, predecesoras de la Central, habían sido legitimadas antes de la llegada de José I por el Gobierno británico, cuyo ministro del Foreign Office, George Canning, había anunciado su apoyo a la causa española en una conocida sesión parlamentaria celebrada en junio de $1808^{17}$. El Reino Unido se preparaba para una nueva política expansionista tras las sucesivas pérdidas territoriales que había sufrido su imperio en América. La entrada de Gran Bretaña en el conflicto espanol le aseguraba un importante papel de orden internacional al lado de una todavía - gran potencia colonial como era la España de principios del siglo XIX. Según afirma Azcárate, la intervención británica tenía también como objetivo inmediato «asegurar la liberación y la defensa de Portugal [...] contra el peligro de la ocupación francesa» ${ }^{18}$. Contando con militares de renombre como sir John Moore (1761-1809), los británicos aportaron ayuda tanto financiera como humana, estableciendo un estrecho contacto con la península ibérica. Ello tendrá como consecuencia la intensificación de las relaciones entre ambos bloques a partir de ese momento, así como la introducción en las islas británicas de un arte y una cultura españolas que hasta entonces habían sido considerablemente limitadas.

El fondo archivístico que contiene los documentos que aquí se presentan se encuentra en The National Archives (Londres). Concretamente, los textos seleccionados han sido extraídos de diferentes legajos pertenecientes a los archivos del War Office (WO), antiguo Ministerio de Defensa británico. La sección WO 1 , entre otras, incluye numerosas series de documentación relativa a la guerra de la independencia. En este caso se ha seleccionado la referente a José I, en particular los textos que incorporan información sobre los bienes conservados en los sitios reales que el monarca francés pudo haberse llevado consigo en el momento de su primera retirada de Madrid en el verano de 1808.

Los documentos reflejan la estupefacción de sus autores ante estos hechos, así como los movimientos de José y de sus tropas durante el desarrollo de la contienda. Las cartas están datadas entre agosto y octubre de 1808, es decir, cuando José Bonaparte ya había salido de Madrid y esperaba la llegada de su hermano para solucionar la caótica situación en que se encontraba. Los autores de estos despachos son el diplomático sir Charles Stuart, el teniente

\footnotetext{
17 Sir Roger Therry, The Speeches of the Right Honourable George Canning, with a memoir of his life, Londres, Ridgway, 1828, vol. 2, págs. 349-353.

18 Pablo de Azcárate, Wellington y España, Madrid, Espasa-Calpe, 1960, pág. 34.
} 
coronel Doyle, los capitanes Cox, Patrick y Carrol y el comandante Roche. Los oficiales, que actuaron como agentes especiales, establecieron lazos muy estrechos con los jefes de los ejércitos españoles: algunos de ellos hablaban castellano y, sin duda, la superioridad militar y financiera del país al que representaban les permitió gozar de una gran influencia en sus decisiones a lo largo de la contienda ${ }^{19}$. Los agentes británicos daban cuenta de sus iniciativas y gestiones tanto al Foreign Office (en calidad de informantes) como al War Office (en calidad de militares). Pronto se distribuyeron en distintas zonas de España. Doyle se situó principalmente en el noreste peninsular (Aragón y Cataluña). Roche se movió durante un tiempo por las provincias vascongadas y Santander y acabó en la zona de Levante al mando de una división española financiada por el Gobierno británico. Carrol, por su parte, estuvo destinado en el noroeste peninsular. Patrick tuvo destinos itinerantes por distintas zonas de la mitad norte de España, y Cox se instaló sobre todo en el sur, principalmente Sevilla y Cádiz. Con un último oficial destinado en Baleares, Whittingham, el Gobierno británico disponía de información fiable acerca de la práctica totalidad del país ${ }^{20}$.

Durante 1808 y 1809, los principales receptores de la información generada por estos agentes fueron lord Castlereagh, al frente del War Office, y el ya mencionado Canning, en el Foreign Office. Castlereagh pasaría a encabezar este último ministerio en 1812, lo que le permitiría poner a prueba sus dotes como gestor durante los años siguientes y, especialmente, en el proceso de restitución de los bienes incautados por Napoleón a lo largo y ancho del continente europeo.

Análisis de la correspondencia enviada a Londres entre agosto y octubre de 1808

El clima que respiraba la corte de Madrid durante este primer año era inevitablemente tenso. La precipitada salida de José I del Palacio Real, que desde hacía varios años había quedado parcialmente abandonado por Carlos IV en favor de otras residencias reales de carácter campestre, dejó tras de sí un ambiente caótico y descontrolado, con el saqueo convertido en práctica habi-

19 Alicia Laspra Rodríguez, «El compromiso británico y la guerra peninsular: diplomacia, milicia y opinión pública», en Conmemoración bicentenario 2 de Mayo de 1808 (ciclo de conferencias, Academia de Artillería, mayo 2008), Segovia, Biblioteca de Ciencia y Artillería, 2008, págs. 121-160 (pág. 151).

20 Poco se sabe hoy en día sobre estos militares y agentes. Recientes estudios intentan compensar estas carencias. Véase por ejemplo la monografía sobre William Parker Carrol de Alicia Laspra y Brian O'Connell, «In bello fortis». La vida del teniente general irlandés sir William Parker Carrol (1776-1842), Oviedo, Fundación Gustavo Bueno, 2009. 


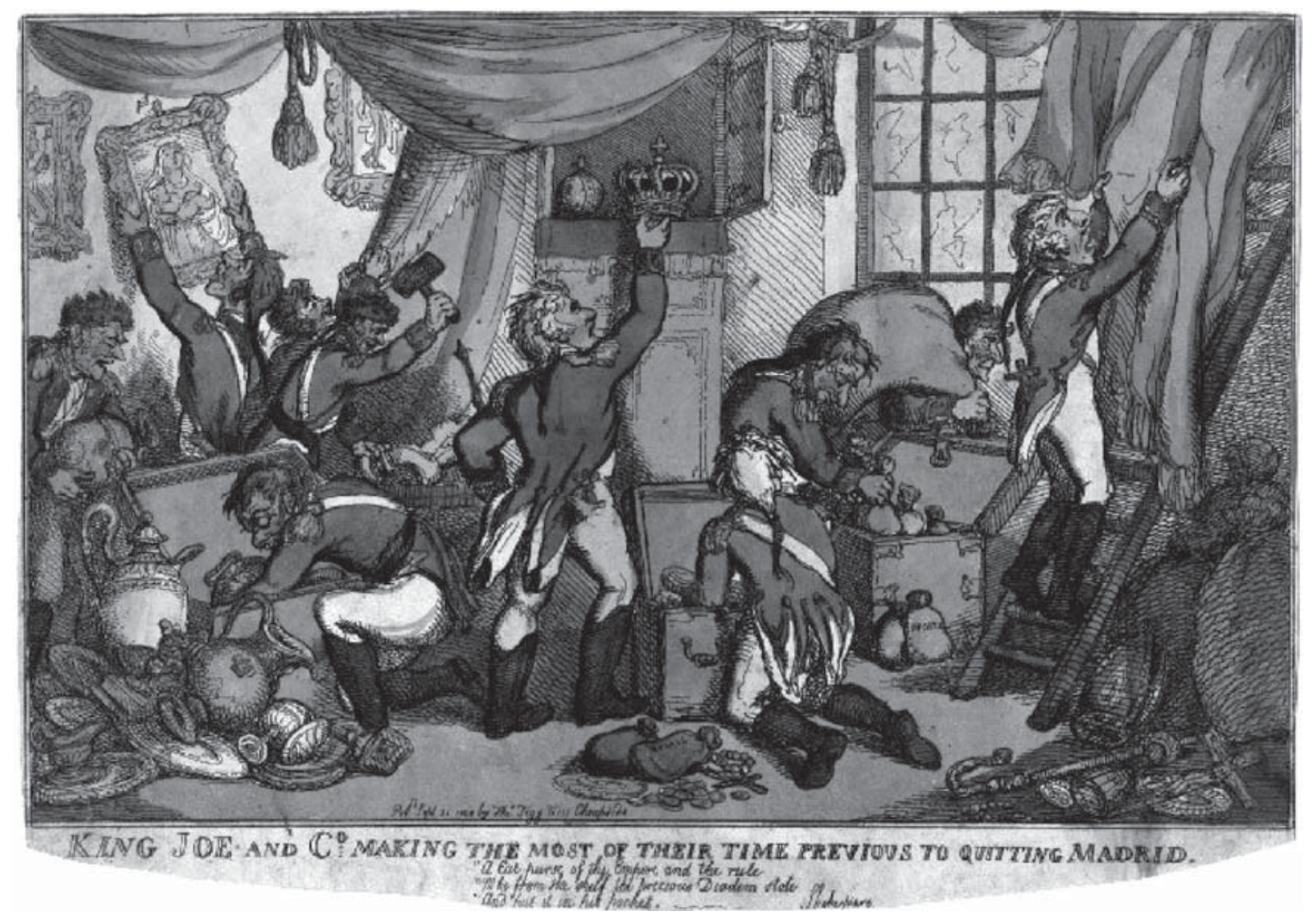

FIG. 1. King Joe and C. ${ }^{\circ}$ making the most of their time previous to quitting Madrid. Autor: Thomas Rowlandson. Editor: Thomas Tegg. Grabado al aguafuerte coloreado a mano. Fecha: 25 de septiembre de 1808. Localización: British Museum, Londres. AN184872001

tual. Según consta en las Mémoires del caballerizo mayor Stanislas Girardin ${ }^{21}$, los oficiales franceses deambulaban por el palacio madrileño a la búsqueda de objetos de valor y armas que pretendían llevarse consigo. Existen varios grabados británicos de 1808 que reflejan este expolio del que habla Girardin. Estas imágenes ${ }^{22}$, que sin duda poseen un toque irónico a la vez que enfático, ilustraron en su día varios episodios de la guerra, actuando a su vez como documentos propagandísticos contra los franceses. En uno de ellos (fig. 1) se representa al propio José con varios oficiales y soldados franceses en una estancia del Palacio Real de Madrid, guardando en sacos y en sus propios bolsillos todos los objetos de valor que son capaces de llevar consigo: una corona, cuadros, una vajilla de

21 Louis Stanislas Cécile Xavier, conde de, Girardin, Mémoires, journal et souvenirs, París, Moutardier, 1829, vol. II, págs. 145-147.

22 Existen varias publicaciones que recogen grabados de este tipo. Véase La alianza de dos monarquías: Wellington en España (cat. expo. Museo Municipal de Madrid, oct.-dic. 1988), Madrid, Fundación Hispano-Británica, 1988; Mark Bryant, Napoleonic Wars in Cartoons, Londres, Grub Street, y Mark Bryant, Dictionary of British cartoonist and caricaturists, 1730-1980, Aldershot, Scolar Press, 1994. 
plata, monedas de oro... A pesar de la buena intención de Girardin de dejar todo tal como lo habían encontrado los franceses a su llegada, las cartas de los agentes británicos también dan fe de que el saqueo fue moneda corriente.

En la primera de estas cartas, fechada el 4 de agosto, Doyle informa a lord Castlereagh de la retirada francesa en dirección a Burgos. La evacuación de Madrid se considera más que probable, teniendo como causa fundamental la «derrota del general Dupont», es decir, la victoria española en la batalla de Bailén. Lo más importante de este documento, en cuanto al expolio, es la afirmación contundente de que «José Bonaparte ha abandonado Madrid llevando consigo todos los objetos de valor que había en la corte». Queda así constancia de las intenciones del rey intruso en el sentido de querer aprovechar su situación privilegiada, por breve que fuese, para hacerse con parte de los bienes de palacio.

La siguiente carta, del 5 de agosto, confirma la retirada francesa de Madrid, e informa de la incertidumbre de la población española, que sigue huyendo a pesar de la salida de los invasores de la capital. Asimismo, se confirma la información que aparecía en la carta anterior, en la que Doyle afirmaba que José salió de la corte acompañado de todos los franceses. Volviendo a las Memorias de Girardin, éste manifiesta que tras supervisar la partida del equipaje del rey, fue el último francés en abandonar Madrid, con lo que se confirma la completa retirada francesa de la capital anunciada por los británicos.

El 8 de agosto, Patrick registra nuevamente la retirada de José y de su séquito, y añade una cita de la Gaceta de Madrid en la que se pide tranquilidad a la población y vigilancia respecto a los peligros que pueda acarrear la falta de unidades militares de defensa en la capital. Según esta información, José estaría en ese momento en Segovia.

La carta escrita desde Gijón a Castlereagh el 12 de agosto de 1808 es una de las más interesantes. Su autor, Roche, sostiene que José ha salido de la capital tras haberse «llevado consigo todo objeto de valor tanto de los palacios reales como de los del Príncipe de la Paz».Y añade que todo ello fue empaquetado y transportado junto con las joyas de la corona y dinero procedente del Banco de Madrid. Por un lado, este testimonio indica que no solamente se intentó sacar obras de arte del país, sino que los franceses también se llevaron dinero perteneciente a los españoles. Por otro lado, además del saqueo perpetrado en los palacios regios, esta carta nos informa de que también el de Buenavista (antaño perteneciente a Manuel Godoy e inicialmente saqueado por Murat ${ }^{23}$ ) se vio

23 María de los Santos García Felguera, Viajeros, eruditos y artistas: los europeos ante la pintura española del Siglo de Oro, Madrid, Alianza, 1991, pág. 50. Sobre la colección Godoy véanse los trabajos de Isadora Rose DE VIEJo, como Manuel Godoy, patrón de las artes y coleccionista, Madrid, UCM, 1983, o «Cuadros 
afectado por el afán expoliador de los invasores. Se revisará más abajo quiénes fueron los protagonistas fundamentales de este expolio.

La siguiente misiva, también del 12 de agosto, contiene información sobre los movimientos de José y de sus tropas. Según Roche, los franceses se encontraban en Palencia, y también disponían de una guarnición en Valladolid. El autor sitúa a José en la primera de estas ciudades, añadiendo que el rey intruso estaba siendo perseguido por el general Castaños. El itinerario continúa hacia el norte del Ebro en dirección a Vitoria.

El 18 de agosto, Roche informa a Castlereagh desde Bilbao de la retirada francesa de Burgos y de que José, que según los rumores se dirigía a la frontera, había sido localizado unos días antes en Vitoria. Esta última afirmación se confirma con la carta siguiente, del 22 de agosto, en la que Patrick informa de que José había pernoctado en Vitoria el día 13 del mismo mes. El documento del día 18 también refleja el desorden que reinaba a la sazón y lo difícil que era para las tropas de la resistencia controlar los movimientos imperiales. Éstos estaban, según Roche, concentrados en Bilbao, y aprovecharon la coyuntura para saquear la ciudad.

El 29 de agosto, Doyle escribe al ministro británico y le habla, por una parte, de la presencia de las fuerzas francesas en Vitoria, hacia donde se dirigen los bienes que fueron expoliados en Madrid. Por otra parte, Doyle confirma la retirada francesa de Burgos, vista la necesidad de provisiones que apremiaba al enemigo. Fueron a buscarlas a la otra orilla del Ebro y añadieron a todo ello las contribuciones exigidas por José a lo largo y ancho de las provincias vascongadas y Navarra.

Ya en septiembre, el día 1, Doyle informa de la casi completa evacuación francesa de Vizcaya, exceptuando Vitoria y San Sebastián. La guardia de José y el propio «rey errante» se dirigen ahora hacia Pamplona, no sin antes haber recibido en Miranda de Ebro el botín proveniente de Bilbao. Logroño, junto con la ciudad castellana, se había convertido definitivamente en un punto estratégico para las tropas ocupantes. También en esta carta se habla de la llegada del botín de Bilbao a Miranda de Ebro. Según la descripción de Doyle, José se tomó muchas molestias para custodiar los 142 carros procedentes de Bilbao, tanto cuando llegaron a la ciudad castellana como cuando salieron de ella.

Las tres últimas cartas pueden analizarse conjuntamente, puesto que en todos los casos se trata de información sobre la posición de José en la ribera norte del río Ebro. Respecto a la primera, del día 5 de septiembre, confirma

de la colección de Manuel Godoy vendidos por la Academia», Boletín de la Real Academia de Bellas Artes de San Fernando (Madrid), núm. 92-93 (2001), págs. 33-44. 
que José se encuentra en Pamplona, desde donde envió su guardia personal hacia otros enclaves controlados por los franceses. En la siguiente misiva, del día 21 de septiembre, Stuart señala que el cuartel general del enemigo estaba situado en Logroño. José había vuelto a desplazarse y lo sitúan en una localidad de la zona. Para finalizar, el 8 de octubre, José aparece en Bilbao, donde aprovecha las facilidades que otorga el puerto vasco para, según Stuart, mejorar su estrategia.

\section{Interpretación de la correspondencia: un primer intento de expolio}

Los datos que aporta la correspondencia que queda resumida se insertan en dos bloques diferenciados a la vez que relacionados entre sí. Por un lado, los autores de estas cartas señalan con pocos días de diferencia el itinerario seguido por José Bonaparte tras su huida de Madrid. Esta información fue sin duda útil en su momento para controlar los movimientos de las tropas francesas y del propio José, constituyendo documentos clave para definir la propia estrategia británica durante esos meses de la guerra. Por otro lado, los agentes y militares británicos indican en varias ocasiones cómo los invasores aprovecharon la coyuntura para sacar objetos de valor de los palacios madrileños. Esta actividad no se ceñiría al primer año del reinado josefino, sino que se convertiría en una práctica recurrente hasta la salida definitiva de los invasores en 1813.

Respecto al saqueo de palacios madrileños, tres de las cartas $(1,4,8)$ recogen información relativa tanto al Palacio Real como al Palacio de Buenavista. A pesar de la innegable tentativa de expolio de 1808, el testimonio de estas misivas debe ser interpretado con precaución. En sólo diez días de estancia en Madrid parece complicado que José haya conseguido empaquetar todas las piezas de valor de los palacios reales, como afirman los documentos primero y cuarto. Además, es necesario tener en cuenta que los agentes británicos informaban de actos que en muchas ocasiones no habían visto con sus propios ojos, con lo que es probable que la versión final de los hechos estuviese ligeramente exagerada. Volviendo al saqueo de estos años, cabría hablar de una persona cuya «misión en España» estuvo ligada al expolio de obras de arte. Se trata de Frédéric Quilliet, personaje calificado de «bastante misterioso» por los especialistas $^{24}$. Quilliet, que actuó como director de Monumentos de España, colaboró

24 Hemel Lipschutz, Spanish painting, pág. 40, n. 70. El análisis más detallado de este personaje data de 1933: Miguel Lasso de la Vega, marqués del Saltillo, Mr. Frédéric Quilliet: comisario de Bellas Artes del Gobierno intruso, 1809-1814, Madrid, Maestre, 1933. Véase también José Luis Sancho, «Francisco de Goya 


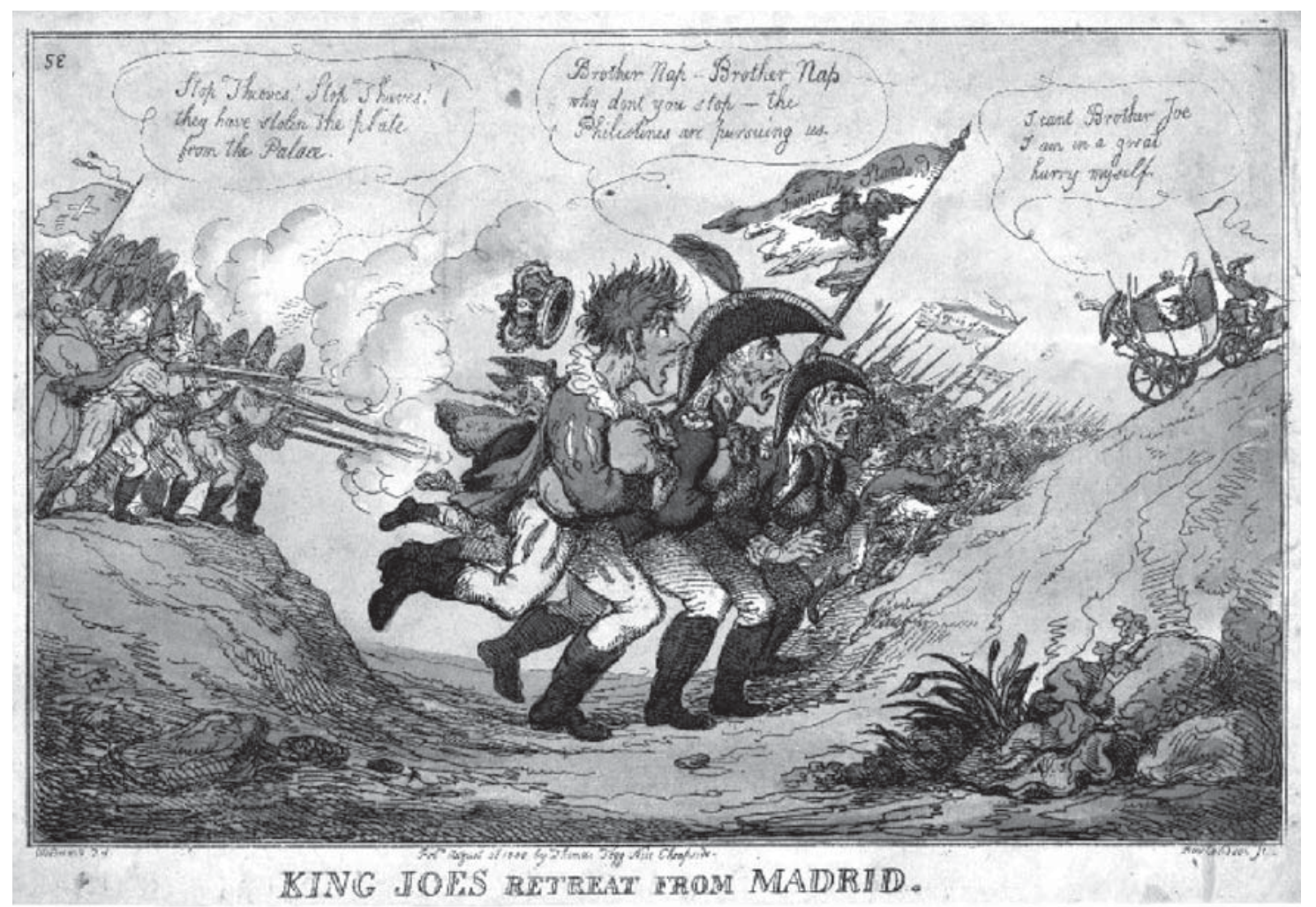

FIG. 2. King Joes retreat from Madrid. Autor: Thomas Rowlandson. Editor: Thomas Tegg. Grabado al aguafuerte coloreado a mano. Fecha: 21 de agosto de 1808. Localización: British Museum, Londres. AN184856001

tanto oficial como extraoficialmente en el saqueo de bienes de la Península. Además de participar en la composición del «obsequio» a Napoleón destinado al museo parisino del emperador —botín de guerra mayoritariamente devuelto tras la caída del corso-, Quilliet mantuvo un estrecho contacto con marchantes de arte como Jean-Baptiste-Pierre Lebrun o August Wallis ${ }^{25}$. Se encontraba en España ya antes de la llegada del propio José Bonaparte, lo que le permitió inspeccionar con antelación las colecciones españolas. En enero de 1808 se encargó de inventariar la colección de Godoy, recientemente confiscada ${ }^{26}$. También fue el autor del inventario de los bienes del Palacio Real en julio de 1808, de manera que José I disponía de un importante resumen de lo que aca-

y Frédéric Quilliet en el Palacio Real de Madrid, 1808», Boletín del Museo del Prado (Madrid), XIX, núm. 37 (2001), págs. 115-142.

25 Hemel Lipschutz, Spanish painting, op. cit., pp. 40-41.

26 Véronique GÉrARd-Powelt, «Les collections des officiers de l'armée impériale pendant la campagne d'Espagne: un butin très varié», en Collections et marché de l'art en France, 1789-1848 (actas de coloquio, París, 2005), Rennes, Presses Universitaires de Rennes, 2005, págs. 305-317 (págs. 306-307). 
baba de «heredar» nada más llegar a la capital de su nuevo reino ${ }^{27}$. El papel de personajes como Quilliet, acusado en 1810 de perpetrar actividades ilícitas en su propio beneficio, fue decisivo a la hora de escoger los bienes que se llevaron los oficiales del ejército napoleónico y el propio José en estos primeros años. No hay constancia de la intervención de Quilliet en el saqueo de 1808, pero lo que sí es seguro es que estaba en España y que conocía el contenido de las colecciones que nutrieron el expolio. Existe un grabado británico de agosto de 1808 (fig. 2) que ilustra tanto la salida precipitada de José de Madrid como el beneficio que de ello obtuvieron los oficiales franceses. En él aparece un grupo de españoles persiguiendo a los «ladrones que han robado la plata del Palacio». Los franceses, que huyen cargados de sacos y con José en primer plano, corren detrás de un carruaje donde se encuentra Napoleón. Éste se niega a esperar por su hermano, cuya corona se desprende de su cabeza.

Se presentan otras dos cartas (6 y 9) que hablan del saqueo, pero esta vez se refieren a los bienes procedentes, no de las colecciones reales, sino de las provincias. A pesar de las dificultades de emprender un estudio detallado sobre el patrimonio español incautado en estos años, gracias a la existencia de inventarios anteriores y posteriores a la guerra, y a los estudios posteriores sobre las colecciones reales y el Museo del Prado ${ }^{28}$, se ha conseguido averiguar el contenido de ciertas extracciones ilícitas procedentes de la capital. Sin embargo, el saqueo en provincias fue también considerable y, exceptuando personajes destacados como Soult y sus incautaciones sevillanas, poco se sabe acerca de estos pillajes. Aun así, mediante esta correspondencia queda constancia del expolio perpetrado en Bilbao, que según Roche fue saqueado por el ejército francés, y del interés personal por parte del rey intruso en asegurar el transporte de los bienes procedentes de la ciudad vasca.

Por lo que respecta al resto de los despachos, lo más relevante de su contenido está ligado al itinerario seguido por las tropas francesas y por el propio monarca tras su salida de Madrid. De todas formas, esta preciosa información plasmada por los británicos no se limita a señalar cuáles fueron los lugares

27 Frédéric Quillet, Description des tableaux du Palais de S. M. C, Real Biblioteca del Palacio Real, II, 3.269, 27 de junio de 1808. Citado por José Luis SANCHO, «Francisco de Goya y Frédéric Quilliet», op. cit., p. 115 y p. 139 n. 1.

28 Sobre los inventarios de estos años contamos con el estudio de Jérôme TrecA, «Les beautés de l’inventaire», Connaissance des Arts (París), núm. 461-462 (jul.-agto. 1990), págs. 68-74, y de Juan José LunA, Pinturas y esculturas del Palacio Real de Madrid en 1811, Madrid, Fundación Rich, 1993. Sobre el Museo del Prado, existen publicaciones clave como la de Mariano de Madrazo, Historia del Museo del Prado: 1818 1868, Madrid, Impr. C. Bermejo, 1945, u otras más recientes de la mano de Alfonso Pérez Sánchez, Pasado, presente y futuro, Madrid, Fundación Juan March, 1977, y de Javier Portús, Museo del Prado. Memoria escrita (1819-1994), Madrid, Museo del Prado, 1994. 
de importancia estratégica para los invasores. Las cartas también describen el clima en que vivía la población (de miedo, de rechazo al invasor, y obligada a pagar contribuciones), indican las fuentes de las que procedían los datos que se notificaban a Londres (generalmente personas directamente relacionadas con la guerra, tanto militares como políticos) y ratifican la estrecha colaboración establecida entre españoles y británicos desde el principio de la contienda (por ejemplo, entre Ballesteros y Roche).

La conexión entre las doce cartas reside en que en ellas también se hace referencia al itinerario que siguió el supuesto botín, reafirmando la existencia de esta práctica desde el año 1808. Según los documentos, los carruajes provenientes de Madrid llegarían a Vitoria un poco más tarde que José, aunque nada se conoce sobre el previsible destino de los mismos. La vuelta de José a Madrid a finales de año pudo haber facilitado la restitución de estos bienes, o también es posible que se hubieran trasladado en parte a Francia desde Vitoria. Estas lagunas son consustanciales a todo capítulo ligado al expolio, dado el movimiento de obras de arte que conllevó esta práctica. En algunos casos, como en el del equipaje del rey José interceptado en la batalla de Vitoria por Wellington en 1813, se dispone de más información ${ }^{29}$, aunque, tal y como ocurre con el expolio de 1808, persisten algunas lagunas respecto al contenido total del botín.

A partir de los datos examinados en este artículo, se presentan muchas cuestiones pendientes de resolver en torno a este episodio de la guerra de la independencia, que podrían considerarse para futuros estudios. En primer lugar, habría que investigar el contenido de estos misteriosos carruajes, dónde y en qué condiciones se almacenaron los objetos de valor y cuál fue su destino final. Estos datos permitirían establecer un vínculo entre el expolio de 1808 y el de 1813, y comprobar si hubo obras de arte que fueron incluidas en los equipajes de ambas ocasiones. En segundo lugar, cabría la posibilidad de profundizar sobre el papel desempeñado por los miembros de la corte que se encontraban en Madrid en el momento de esta primera huida, a la hora de seleccionar y transportar las obras de arte. Se trata tanto de Quilliet como de otros posibles colaboradores. En último lugar, el enigmático papel ejercido por José Bonaparte respecto al patrimonio nacional plantea interrogantes acerca del antiguo monarca y su relación con el arte. Es curioso observar cómo, por una parte, José tuvo verdaderas iniciativas para promover la cultura y la preservación del patrimonio (entre otras con los decretos que prohibían la salida del país de

29 Respecto al saqueo de 1813, además de haber sido estudiado por la mayoría de los autores citados, añadimos el recientemente reeditado catálogo de Apsley House: Susan Jenkins (ed.), Catalogue of paintings in the Wellington Museum Apsley House, Londres, English Heritage \& Paul Holberton, 2009. 


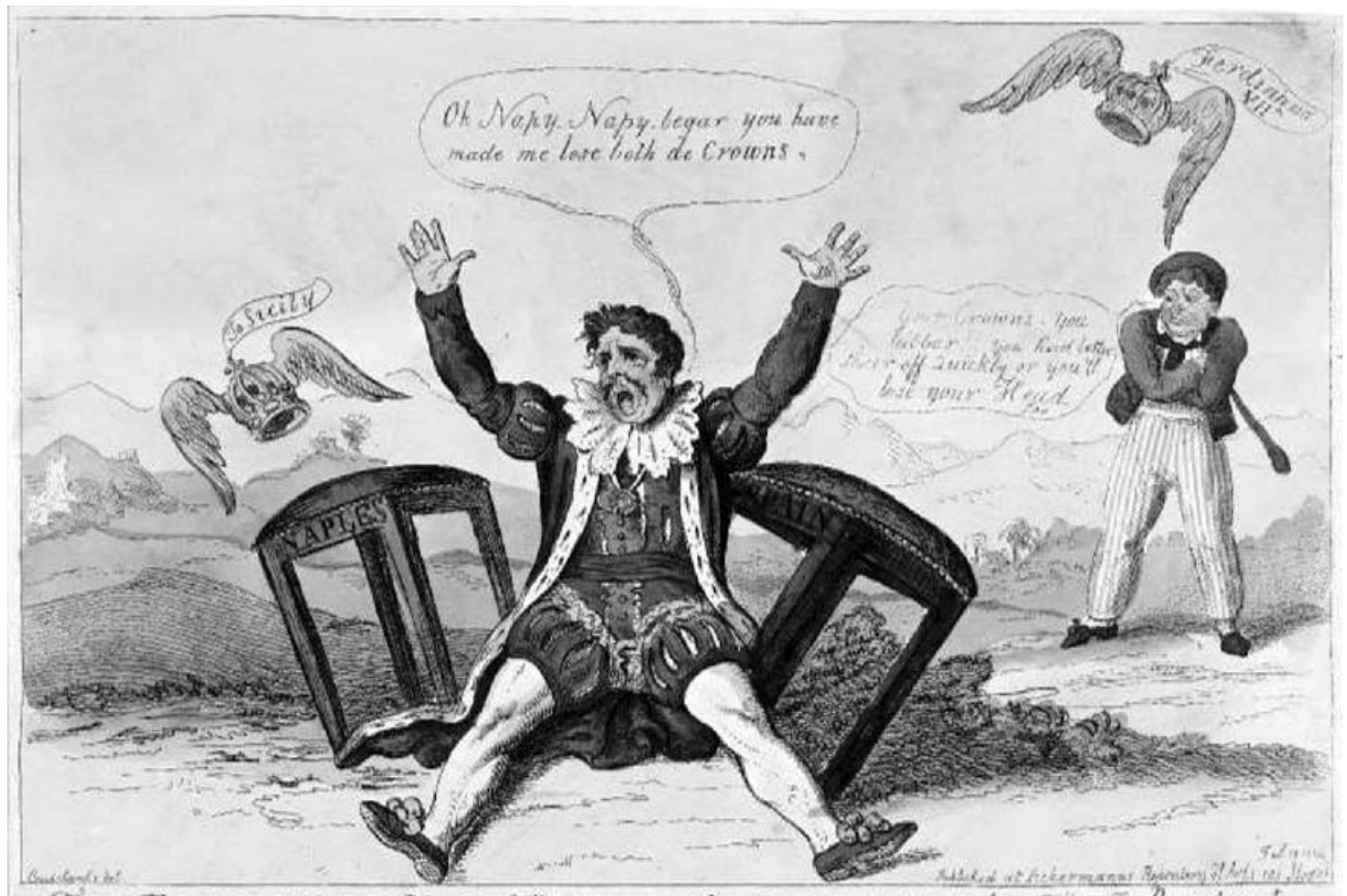

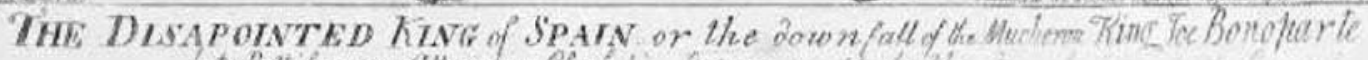

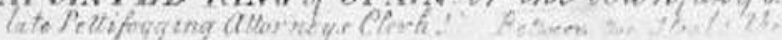

FIG. 4. The disappointed King of Spain - or the downfall of the Mucheron King Joe Bonoparte late pettifogging attorneys clerk! Autor: Isaac Cruikshank. Editor: Ackermann. Grabado al aguafuerte coloreado a mano. Fecha: 19 de julio de 1808. Localización: British Museum, Londres. AN539692001

obras de arte y la fundación del futuro Museo del Prado) y cómo, por otro lado, aprovechó, tanto en 1808 como en 1813, para intentar beneficiarse él mismo del saqueo. Puede, finalmente, que una de las razones que se han imputado al pillaje sea cierta: que «solamente un hombre roto moralmente y con los ideales perdidos pudo dedicarse a tan incalificable expoliación» ${ }^{30}$. De nuevo otro grabado de 1808 (fig. 4) ilustra esta afirmación mediante la representación del rey José en una actitud abatida y desesperada, al verse privado tanto de la corona de Nápoles como de la española. Fue éste sin duda un reinado complicado, del que, sin embargo, se beneficiaron muchas más personas de lo que se conoce hasta hoy en día.

30 Juan José Luna, «El Rey José», en La alianza, pág. 227. 
Apéndice: extractos de documentos conservados en los archivos del War Office

1

1808, agosto, 4, La Coruña. Teniente coronel Charles Doyle a lord Castlereagh. WO 1/227, f. 115.

I have this moment had a letter dated Madrid $27^{\text {th }}$ July received by one of this Junta from his nephew; who states that in the morning of that day the French had commenced their retreat, and he believed that the next day, «their dear friends would have completely evacuated the capital».

He attributes this sudden movement to the defeat of General Dupont.

«Every French man whatever belonging to the Court or not, is following the army».

«Joseph Buonaparte has quit Madrid and has taken with him everything of value belonging to the Court».

«The French are retiring in the direction of Burgos». [...]

I confess, my Lord, I have no doubt upon my mind as to the truth of the evacuation of Madrid; the report has come from many different quarters, and it now comes from the spot itself. [...]

\section{2}

1808, agosto, 5, Sevilla. Capitán William Cox a lord Collingwood. WO 1/231, f. 313 .

[...] Joseph Bonaparte has undoubtedly left Madrid, accompanied by several of the grandees, and all the ministers, expect Cevallos. The inhabitants are in the greatest consternation and are flying from the capital en great numbers into this and the adjacent provinces, as the only places of security. [...]

3

1808, agosto, 8, Oviedo. Capitán Patrick a lord Castlereagh. WO 1/233, ff. 562 y 570 .

[...] I have seen various private letters from Madrid dated $30^{\text {th }}$ ultimo, informing that Joseph Bonaparte was there proclaimed on the $25^{\text {th }}$, but with every expression of disgust on the part of the people. 
It is strongly reported, and credited by many persons of consequence (although I cannot trace it to any substantial source) that he has quitted Madrid with his army and retired to Segovia. [...]

P. S. I have the outmost satisfaction in adding for your Lordship's information that gazettes have just arrived from Madrid, dated $1^{\text {st }}$ August (one of which I have seen), exhorting the inhabitants to tranquillity, as the French troops being all gone and the city without garrison, evil minded people might take the occasion to commit disorder. [...]

1808, agosto, 12, Gijón. Comandante Roche a lord Castlereagh. WO 1/233, ff. 459, 463-464.

My Lord,

Excesses and events, of the greatest magnitude, are rapidly following one another in this country. In the letter I had the honour of addressing to your Lordship by his Majesty's ship Comet on the $9^{\text {th }}$ instant your Lordship will receive the important intelligence of the defeat and surrender of the French army, under General Dupont, near Andújar in Andalucía and of the total evacuation of Madrid on the $1^{\text {st }}$ instant by the Enemy, with the exception of 2,500 sick and wounded left in the hospitals. [...]

The flight of Joseph Bonaparte and the French Army from Madrid was marked by circumstances if possible more strongly characteristic of the rapine and plunder by which their route through Spain may be traced. [...]

Joseph set off on horseback surrounded by a French column on the $30^{\text {th }}[\ldots]$ however, every article of any value as well in the Royal Palaces as in those of the Prince of Peace, were packed up and carried off. Among the first ones the jewels of the Crown, and a contribution of two hundred thousand pounds levied on the Bank of Madrid. Every horse and mule in the capital was put in requisition to convey as well their plunder and baggage as the light guns in the Buen Retiro, at which place they spiked 100 guns on large calibre which they could not carry off. $[\ldots]$ 
1808, agosto, 12, Gijón. Comandante Roche a lord Castlereagh. WO 1/233, ff. $482-483$.

[...] General Ballesteros whom I had a long conversation with, and with whom I have agreed to keep up constant correspondence while I remain at Bilbao and Santander) confirms the intelligence that Palencia is to be occupied in force, by the French where, it is also added, Joseph Bonaparte has arrived. They have also a small garrison at Valladolid -at either of which places I can not conceive they think of making any stand- and indeed their situation appears to be extremely hazardous.

Castaños being, from the last accounts we can collect, by this time to the Northward and Eastward of Madrid in pursuit of Joseph Bonaparte's army which fled from the capital. [...]

6

1808, agosto, 18, a bordo del buque de S. M. Seine, en aguas de Bilbao. Comandante Roche a lord Castlereagh. WO 1/233, ff. 500-501.

[...] It appears My Lord that the French Corps now in Bilbao came from Victoria [sic] where Joseph Bonaparte passed through as it is reported in the most positive manner 3 or 4 days ago by a variety of people who I closely questioned on the subject and they all say he passed though on his way into France whither the whole of the French army is retiring -it is impossible my Lord to track for or speak with certainty of any of the reports we hear- but the intelligence we have got from them states the French have certainly left Burgos and that this Corps now plundering Bilbao is a part of the rear guard of the whole army, and of course cannot remain more than 2 or 3 days. [...]

1808, agosto, 22, Oviedo. Capitán Patrick a lord Castlereagh. WO 1/233, f. 593.

[...] The Major [Roche] had been assured that Joseph Bonaparte slept at Vitoria on the $13^{\text {th }}$ instant.

It is reported that the enemy has abandoned Burgos. [...] 
1808, agosto, 29, Madrid (por la noche). Teniente coronel Charles Doyle a lord Castlereagh. WO 1/227, 241-242.

[...] A deputy from Navarra arrived here yesterday; he stated the zeal and patriotism of the people but their total incapacity to act as they are overun by the French - he states their numbers to be about 40,000 Infantry and 6,000 Dragoons-. The plunder of Madrid is still upon the road near Victoria [sic] and Joseph Buonaparte levying contributions all through Biscay and Navarra and particularly in the neighbourhood of Burgos, from which town the French have decidedly only retired in order to take up positions for the sake of provisioning their army. They were in great want of provisions at Burgos. [...]

1808, septiembre, 1, Madrid. Teniente coronel Charles Doyle a lord Castlereagh. WO 1/227, ff. 263-264 y 266.

He [a deputy from Álava] declares the whole of Biscay to be evacuated except Vittoria and St. Sebastian. The whole of the troops posted between Miranda del Ebro and Logroño passed through the latter town upon the 29th August to the number of 16,000, in which Joseph Buoparte's guard of near 4,000 men is included, which guard together with the wandering king proceeded to Pamplona; the remainder of the troops filed off to right along the Ebro. ...

Joseph Buonaparte declares 20,000 men are on their way from France to join him but we cannot learn that one single soldier started the march in that country. $[\ldots]$

[P. S.] Joseph Buonaparte waited at Miranda the arrival of 142 carts laden with the plunder of Bilbao. He literally rode out to meet them and accompanied them back out of the town. [...]

10

1808, septiembre, 5, Madrid. Teniente coronel Charles Doyle a lord Castlereagh. WO 1/227, f. 289.

A confidencial person whom I employed at Salamanca to obtain intelligence for me has this moment arrived, having passed within the last ten days through 
the whole chain of French posts, and the day before yesterday left Borja, near Zaragoza, where 24,000 French were assembled, of whom 2,000 Dragoons, Joseph having brought down his guard from Pamplona to join generals Lefebre and Moncey. [...]

1808, 21, septiembre, S/l. Charles Stuart a Wilson. WO 1/230, f. 281.

[...] The French in the Rioja have their headquarters at Logroño. Joseph was lately at $[\ldots]$ but they are not alarmed for his principal army. [...]

1808, octubre, 8, Balmaseda, cuartel general del ejército de Galicia. Capitán William Parker Carrol a lord Castlereagh. WO 1/231, f. 434.

[...] Joseph Buonaparte is at present at Bilbao, and appears to be most perfectly aware of the very great importance of which the possession of that port is to the security of the French armies. [...]

Apéndice: traducción al español de los extractos de los documentos citados

1

1808, agosto, 4, La Coruña. Teniente coronel Charles Doyle a lord Castlereagh. WO 1/227, f. 115.

Acabo de leer una carta fechada el 27 de julio en Madrid recibida por uno de los miembros de la Junta y procedente de un sobrino suyo. Éste expone que, en la mañana de ese día, los franceses habían iniciado su retirada y creía que al día siguiente «sus queridos amigos habrían evacuado al completo la capital». Atribuye este repentino movimiento a la derrota del general Dupont.

«Todos los franceses, pertenecientes o no a la corte, acompañan al ejército».

«José Bonaparte ha abandonado Madrid llevando consigo todos los objetos de valor que había en la corte». 
«Los franceses se están retirando en dirección a Burgos».

Debo confesar, señor, que no tengo dudas acerca de veracidad de la evacuación de Madrid. La misma información nos ha venido desde distintos lugares, y ahora procede de la propia capital.

2

1808, agosto, 5, Sevilla. Capitán William Cox a lord Collingwood. WO 1/231, f. 313 .

No hay duda de que José Bonaparte ha abandonado Madrid acompañado de varios aristócratas y de todos los ministros, con la excepción de Cevallos. Los habitantes están consternados y huyen de la capital en masa hacia esta provincia y hacia otras cercanas, por ser los únicos lugares seguros.

1808, agosto, 8, Oviedo. Capitán Patrick a lord Castlereagh. WO 1/233, ff. 562 y 570 .

He leído varias cartas privadas procedentes de Madrid, fechadas del día 30 del pasado mes, informando de que José Bonaparte fue proclamado allí el día 25 , pero con todo tipo de rechazo por parte del pueblo.

Muchas personas fiables (aunque no puedo citar una fuente sustancial) informan de manera contundente y acreditan que ha abandonado Madrid con su ejército y se ha retirado a Segovia.

P. D. Acaban de llegar gacetas de Madrid fechadas el 1 de agosto (una de las cuales he visto) exhortando a los habitantes a la tranquilidad dado que todas las tropas francesas se han ido y como la ciudad carece de guarnición, personas malvadas podrían aprovechar la ocasión para cometer desórdenes.

1808, agosto, 12, Gijón. Comandante Roche a lord Castlereagh. WO 1/233, ff. $459,463-464$.

Una serie de excesos y acontecimientos de gran importancia se suceden muy deprisa en este país. En la carta que tuve el honor de enviar a su ilustrísima 
por el buque de su Majestad, el Comet, el día 9 del presente mes, se le informa de la derrota y rendición del ejército francés, bajo las órdenes del general DuPont, cerca de Andújar en Andalucía, así como acerca de la evacuación completa de la ciudad de Madrid por parte del enemigo el día 1, con la excepción de 2.500 enfermos y heridos que se encuentran en los hospitales.

La huida de Madrid por parte de José Bonaparte y del ejército francés ha estado marcada por unos hechos que caracterizan, si ello fuera posible, del modo más contundente, la rapiña y el saqueo que se han venido observando en España. José partió a caballo rodeado de una columna francesa el día 30 [...], prepararon y sacaron todo objeto de valor tanto de los palacios reales como de los del Príncipe de la Paz. Entre los primeros estaban las joyas de la corona y un total de doscientas mil libras extraídas del Banco de Madrid. Todos los caballos y las mulas de la capital se requisaron para usarse como transporte, tanto del botín y el equipaje como de la artillería ligera instalada en el Buen Retiro, en donde destruyeron cien cañones de gran calibre que no podían transportar.

1808, agosto, 12, Gijón. Comandante Roche a lord Castlereagh. WO 1/233, ff. $482-483$.

El general Ballesteros (con quien mantuve una larga conversación y con quien he acordado intercambiar correspondencia de manera constante mientras permanezca en Bilbao y Santander) confirma información según la cual los franceses ocuparán Palencia por la fuerza, y añade que José Bonaparte ha llegado a esta ciudad. Tienen también una pequeña guarnición en Valladolid —no puedo saber en cuál de estos dos lugares piensan fijar su posición- y verdaderamente su situación parece ser extremadamente peligrosa. Castaños, según las últimas noticias que nos han llegado, se encuentra en este momento entre el norte y el este de Madrid, tras los pasos del ejército de José Bonaparte que salió a toda prisa de la capital.

6

1808, agosto, 18, a bordo del buque de S. M. Seine, en aguas de Bilbao. Comandante Roche a lord Castlereagh. WO 1/233, ff. 500-501.

Parece ser, milord, que las tropas francesas que se encuentran ahora en Bilbao procedían de Vitoria, por donde pasó José Bonaparte hace tres o cuatro 
días, según informan de manera fehaciente varias personas a las que interrogué personalmente sobre el asunto, y todos dicen que cruzó Vitoria a su paso hacia Francia y, por el momento, la totalidad del ejército francés se está retirando. Es imposible, milord, rastrear o comentar con certeza los informes que nos llegan, según los cuales se indica además que los franceses han abandonado Burgos con toda seguridad, y que las fuerzas que están saqueando Bilbao pertenecen, en parte, a la retaguardia del ejército y, naturalmente, no pueden quedarse durante más de dos o tres días.

1808, agosto, 22, Oviedo. Capitán Patrick a lord Castlereagh. WO 1/233, f. 593.

Han asegurado al comandante [Roche] que José Bonaparte pasó la noche en Vitoria el día 13 del presente mes.

Se informa de que el enemigo ha abandonado Burgos.

1808, agosto, 29, Madrid (por la noche). Teniente coronel Charles Doyle a lord Castlereagh. WO 1/227, ff. 241-242.

Un diputado de Navarra llegó aquí ayer y dejó constancia del entusiasmo y patriotismo del pueblo, que sin embargo está totalmente incapacitado para la acción al haber sido arrollado por los franceses. El producto del saqueo de Madrid está aún de camino cerca de Vitoria, y José Bonaparte exigiendo contribuciones por toda Vizcaya y Navarra, y de forma especial en las cercanías de Burgos, de cuya ciudad los franceses se han retirado con toda seguridad para tomar posiciones encaminadas a la obtención de provisiones para su ejército. Tenían gran necesidad de provisiones en Burgos.

\section{9}

1808, septiembre, 1, Madrid. Teniente coronel Charles Doyle a lord Castlereagh. WO 1/227, ff. 263-264 y 266.

Él [un diputado de Álava] ha declarado que toda Vizcaya ha sido evacuada, con excepción de Vitoria y San Sebastián. Todas las tropas apostadas entre Mi- 
randa de Ebro y Logroño pasaron por esta última ciudad el 29 de agosto, con un contingente de 16.000 hombres, incluyendo la guardia de José Bonaparte, que está compuesta por 4.000 hombres. Dicha guardia, junto con el rey errante, se dirigía hacia Pamplona.

José Bonaparte ha manifestado que han partido 20.000 hombres desde Francia para unirse a él, aunque no hay noticias de que un solo soldado haya iniciado la marcha desde ese país.

[P. D.] José Bonaparte esperó en Miranda la llegada de 142 carros cargados con el botín de Bilbao. Literalmente salió cabalgando a su encuentro y los acompañó de nuevo cuando salían de la ciudad.

1808, septiembre, 5, Madrid. Teniente coronel Charles Doyle a lord Castlereagh. WO 1/227, f. 289.

Acaba de llegar una persona de confianza que había enviado a Salamanca a fin de obtener información para mí, tras haber atravesado durante estos últimos días toda la cadena de puestos franceses y haber dejado anteayer Borja, cerca de Zaragoza, donde se encontraban agrupados 24.000 franceses, incluidos 2.000 dragones. José Bonaparte había enviado su guardia desde Pamplona para unirse a los generales Lefebvre y Moncey.

1808, 21, septiembre, S/l. Charles Stuart a Wilson. WO 1/230, f. 281.

Los franceses situados en La Rioja tienen su cuartel general en Logroño. José estuvo recientemente en [...], pero no están preocupados en exceso por el grueso de su ejército.

1808, octubre, 8, Balmaseda, cuartel general del ejército de Galicia. Capitán William Parker Carrol a lord Castlereagh. WO 1/231, f. 435.

José Bonaparte se encuentra en Bilbao en este momento, y parece ser muy consciente de la importancia estratégica de este puerto para la seguridad de los ejércitos franceses. 